\title{
Combined diagnosis of breast cancer in the early stage by MRI and detection of gene expression
}

\author{
DENA KE, RONG YANG and LINA JING \\ Radiology Department, Beijing Tiantan Hospital, Capital Medical University, Beijing 100050, P.R. China
}

Received August 18, 2017; Accepted January 26, 2018

DOI: $10.3892 /$ etm.2018.6242

\begin{abstract}
Breast cancer is one of the most common cancer types in humans. Magnetic resonance imaging (MRI) is an efficient method for the detection of human breast cancer. However, the efficacy of MRI in detecting breast cancer in the early stage requires to be improved. The present study investigated the diagnostic efficacy of a combination of MRI and detection of gene expression in patients with breast cancer in the early stage. The gene expression levels of Ki-67, BCL11A, FOXC1, HOXD13, PCDHGB7 and her-2 were used as an auxiliary diagnostic index for patients with breast cancer in the early stage. Higher expression levels of TPA and C2erbB22 were observed in tumor tissue obtained from diagnostic biopsy and determined by immunohistochemistry, which indicated a higher risk of breast cancer in a total of 84 participants. Diagnostic data revealed that combination MRI and detection of gene expression had a significantly higher diagnostic rate (66/84) in diagnosing breast cancer in an early stage compared with either MRI (78/360) or detection of gene expression $(72 / 84 ; \mathrm{P}<0.01)$. It was indicated that the combination of MRI and detection of gene expression had a higher diagnostic rate $(94.5 \%)$ than either MRI $(81.4 \%)$ or detection of gene expression $(75.5 \%)$. Histological analysis confirmed the diagnosis determined by MRI and detection of gene expression. These results suggest that the combination of MRI and detection of gene expression may be a potential diagnostic method for assessing patients with early-stage breast cancer.
\end{abstract}

\section{Introduction}

Breast cancer is one of the most common gynecological tumor types worldwide (1). The majority of breast cancer-associated mortalities is caused by local migration and distant metastases of tumor cells (2). While the current therapeutic schedules, including surgical techniques, as well as radiation,

Correspondence to: Professor Lina Jing, Radiology Department, Beijing Tiantan Hospital, Capital Medical University, 6 Tiantan Xili, Dongcheng, Beijing 100050, P.R. China

E-mail: jinglinabeijing@outlook.com

Key words: breast cancer, gene expression, detection, magnetic resonance imaging, early diagnosis chemotherapy and gene targeted therapy, have improved the overall survival (OS) of breast cancer patients, the outcome remains generally poor $(3,4)$. Of note, previous studies have indicated that numerous genes are involved in epigenetic modifications among patients with breast cancer (5-7). Genetic analyses and testing for inherited gene mutations in patients with breast cancer have been reported in clinical studies $(8,9)$. Therefore, it is essential to explore potential genes for diagnosing patients with breast cancer in the early stage.

At present, ultrasound, fluorodeoxyglucose-positron emission tomography (PET)/computed tomography (CT) and magnetic resonance imaging (MRI) are widely used for diagnosing and staging of human breast cancer (10-12). Eminently, MRI provides a higher sensitivity and accuracy in the detection of breast cancer than CT and ultrasound (13). Application of dynamic contrast enhancement MRI and post-processing techniques are helpful for making the correct diagnosis for patients with suspected breast cancer (14). Youk et al (15) have suggested that MRI may be used to identify malignant breast lesions by analyzing their morphological and kinetic features. In addition, MRI is more efficient than CT in assessing the response to neo-adjuvant chemotherapy and is beneficial for identifying the primary tumor in breast cancer patients (16). Furthermore, comparison between PET/CT and MRI in the diagnosis, staging and follow-up of breast cancer patients revealed that MRI is useful for distinguishing between benign and malignant pulmonary nodules, has a high sensitivity and specificity for nodal staging, and is helpful for evaluating the early response to systemic chemotherapy (17). However, the diagnostic accuracy of single MRI for breast cancer is insufficient (18). In recent years, genetic diagnosis of breast cancer has been applied, which may be an accurate and novel diagnostic method for the evaluation of sentinel lymph node metastasis in breast cancer patients (19-21).

In the present study, the diagnostic efficacy of MRI in combination with detection of gene expression in breast cancer patients was evaluated. It was reported that MRI combined with detection of gene expression not only improves the accuracy, but also contributes to the selection of efficient treatments for patients with breast cancer.

\section{Materials and methods}

Patients and selection. A total of 84 female patients (median age, 46.2 years; range, 28.4-65.5 years) with suspected breast 
cancer who were assessed at the Radiology Department of Beijing Tiantan Hospital (Beijing, China) between May 2015 and October 2016 were recruited for the present study. All patients underwent pre-operative MRI and/or detection of gene expression (Ki-67, BCL11A, FOXC1, HOXD13, PCDHGB7 and her-2). All patients were finally diagnosed by histopathology in diagnostic biopsy samples. Patients with a family history of cancer, chronic renal failure and heart disease, as well as those with a history of ipsilateral breast surgery, chest radiotherapy and oncoplastic breast cancer were excluded from the study. Patients who received neoadjuvant chemotherapy or radiotherapy were also excluded from the recruitment. The present study was approved by the Ethics Committee of Capital Medical University (Beijing, China). All patients provided written informed consent.

MRI analysis. The MRI protocol was in accordance with that described in a previous study (22). In brief, a Siemens Verio 3.0T magnet MRI machine (Siemens AG, Munich, Germany) was used to analyze breast tumor lesions and determine their volume. All patients with suspected breast cancer were subjected to MRI screening. MR images were analyzed using a SUN T2000 workstation (Sun Microsystems Inc., Mountain View, CA, USA) with the Eigentool image analysis software 3.0 (Image Analysis Lab, Henry Ford Hospital, Detroit, MI, USA). Threshold ranges were determined using histogram analysis from regions of interest placed around the identified lesion using $95 \%$ confidence intervals determined from signal intensity.

Detection of gene expression. Total RNA was extracted from tumor cells $(1.0 \mu \mathrm{g})$ in biopsy samples using an RNeasy Mini kit (Qiagen, Hilden, Germany) according to the manufacturer's protocol. Total RNA $(1 \mu \mathrm{g})$ was reverse transcribed into complementary (c)DNA using a QuantiTect Reverse Transcription kit (cat. no. 205310; Qiagen) according to the manufacturer's protocol. The cDNA (10 ng) was subjected to quantitative polymerase chain reaction analysis using SYBR Green Master mix (Bio-Rad Laboratories, Inc., Hercules, CA, USA). All primers were synthesized by Invitrogen (Thermo Fisher Scientific, Inc., Waltham, MA, USA) as described previously (23). The reaction conditions were accordance with those of a previous study (24). Relative mRNA expression changes were calculated using the $2^{-\Delta \Delta \mathrm{Cq}}$ method (25). The results are expressed as a fold of the control.

Histopathology. Breast tumor staging was performed based on the American Joint Committee on Cancer Staging manual, sixth edition (26). Histological analysis was performed using the modified Bloom-Richardson classification (27). Primary breast cancer tissues were evaluated from formalin-fixed, paraffin-embedded tumor sections using immunohistochemistry. Tumor sections were incubated with primary antibodies rabbit anti-human against estrogen receptor (ER; 1:1,000; cat. no. clone SP1; Neomarkers for Lab Vision, Fremont, CA, USA) and progesterone receptor (1:1,000, PR; cat. no. clone PgR 636; Dako, Glostrup, Denmark) for $12 \mathrm{~h}$ at $4^{\circ} \mathrm{C}$. Tumor tissues were then incubated with using horseradish peroxidase-conjugated goat anti-rabbit immunoglobulin $\mathrm{G}$ monoclonal antibody (1:1,000, cat. no. PV-6001; Zhongshan Goldenbridge-BIO, Beijing, China) for $2 \mathrm{~h}$ at $37^{\circ} \mathrm{C}$. A Ventana Benchmark automated staining system (Olympus BX51, Olympus; Tokyo, Japan) was used to assess protein expression in tumor tissues. The staining results were semi-quantitatively evaluated by multiplying the staining intensity and the percentage of positively stained cells (magnification, $\mathrm{x} 400$ ). The cutoff value for ER and PR positivity was $>10 \%$ staining on immunohistochemistry (27).

Treatment. Standard treatments for patients with breast cancer in the present study were in accordance with those described in a previous study (28). In brief, breast cancer patients received breast surgery and breast radiotherapy.

Statistical analysis. Values are expressed as the mean \pm standard deviation of triplicate experiments. Pearson's correlation analysis was performed to assess correlations. All data were analyzed with SPSS version 20 (IBM Corp., Armonk, NY, USA) using one-way analysis of variance followed by Tukey's multiple comparison post-hoc test. The OS rates were calculated using the Kaplan-Meier method and the log-rank test. $\mathrm{P}<0.05$ was considered to indicate a statistically significant difference.

\section{Results}

MRI diagnosis of patients with breast cancer. MRI was used to diagnose patients with suspected breast cancer in a total of 84 cases. In a total of 78 patients, a breast lump was detected, which required further confirmation by pathological analysis. Representative MRI images of breast lumps in patients with suspected breast cancer are displayed in Fig. 1.

Analysis of breast cancer-associated gene expression in biopsy samples of patients with breast cancer. To identify differences in gene expression in patients with breast cancer, the gene expression levels of Ki-67, B-cell CLL/lymphoma 11a (BCL11A), forkhead box (FOX)C1, homeobox (HOX) D13; protocadherin $\gamma$ subfamily B, 7 (PCDHGB7) and her-2 were detected in patients with breast cancer. The analysis identified 72 patients with elevated gene expression of Ki-67, BCL11A, FOXC1, HOXD13, PCDHGB7 and her-2, while 32 patients had lower expression (Table I). Of the 78 patients in which a breast lump was detected on MRI, 72 had higher gene expression. The present study demonstrated that there were a total of 66 patients with and 12 patients without breast tumors (Table I).

Pathological analysis of breast cancer tissue. Histopathological analysis was used to confirm whether patients had breast cancer. Analyses demonstrated that all cancer tissues were either non-infiltrative carcinoma or early invasive carcinoma. Representative images of non-cancerous lump, as well as non-infiltrative carcinoma and invasive carcinoma tissue sections are displayed in Fig. 2. A total of 66 patients were finally diagnosed with breast cancer, including 45 cases of non-infiltrative carcinomas and 21 cases of invasive carcinoma (Table II). 

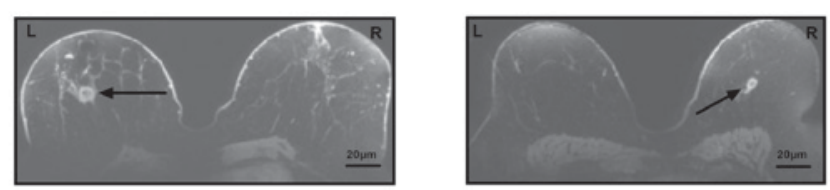

Figure 1. Diagnosis of breast cancer by magnetic resonance imaging. Representative images of breast cancer tissue. (A) Tumor lesion (arrow) in the left breast. (B) Tumor lesion (arrow) in the right breast (magnification, $\mathrm{x} 0.25)$. Scale bar, $20 \mu \mathrm{m}$. L, left breast; R, right breast.

Efficacy of combined diagnosis of MRI and detection of gene expression for patients with breast cancer. The efficacy of combined diagnosis of MRI and detection of gene expression was evaluated in patients with breast cancer. Pearson's R test was used to assess the correlation between gene expression and the risk of breast cancer. A significant positive correlation was observed between expression levels of the genes Ki-67, BCL11A, FOXC1, HOXD13, PCDHGB7 and her-2, and the risk of breast cancer (Pearson's $\mathrm{R}=0.516$; $\mathrm{P}<0.01$; Fig. 3 ). In addition, a significant positive correlation was observed between gene expression levels and tumor size in patients with breast cancer (Pearson's $\mathrm{R}=0.410 ; \mathrm{P}<0.01$; Fig. 4). It was demonstrated that the efficacy of combined diagnosis by MRI and detection of gene expression improved the diagnostic rate compared with that achieved by either MRI or detection of gene expression alone (Fig. 5).

Association between gene expression levels and breast cancer subtypes. The present study then analyzed the association between gene expression levels and the breast cancer subtype, including non-infiltrative carcinoma and early invasive carcinoma. As presented in Fig. 6, breast tumor-associated genes were lower expressed in non-infiltrative carcinoma compared with early invasive carcinoma.

Survival of breast cancer patients diagnosed by MRI and detection of gene expression. The survival of breast cancer patients diagnosed by MRI and detection of gene expression enrolled in the present study was then investigated. A significant improvement of patients' survival time was observed after diagnosis by MRI and detection of gene expression compared with the mean survival of breast cancer patients diagnosed by MRI and detection of gene expression (Fig. 7). These outcomes suggest that diagnosis with a combination of MRI and detection of gene expression contributes to the early implementation of treatment and therefore patient survival.

\section{Discussion}

The efficacy of contrast-enhanced MRI in the diagnosis of breast cancer has been previously proven (29). In addition, diagnosis of breast cancer using malignancy-associated biomarkers has been widely accepted (30). The present study analyzed the efficacy of the combination of MRI and detection of gene expression in diagnosing patients with breast cancer in the early stage. The results indicated that combination of MRI and detection of gene expression not only markedly improved the diagnostic accuracy, but also contributed to early tumor
Table I. Gene expression in patients with suspected breast cancer.

\begin{tabular}{lcc}
\hline Gene & $\begin{array}{c}\text { Patients with breast } \\
\text { tumors }(\mathrm{n}=66 ; \%)\end{array}$ & $\begin{array}{c}\text { Individuals without } \\
\text { tumors }(\mathrm{n}=12 ; \%)\end{array}$ \\
\hline Ki-67 & $9.74 \pm 2.47$ & $2.2 \pm 1.2$ \\
BCL11A & $16.2 \pm 4.2$ & $1.8 \pm 0.7$ \\
FOXC1 & $8.4 \pm 3.2$ & $2.3 \pm 1.0$ \\
HOXD13 & $7.5 \pm 3.0$ & $2.6 \pm 1.5$ \\
PCDHGB7 & $6.8 \pm 2.6$ & $1.7 \pm 0.7$ \\
Her-2 & $9.3 \pm 3.5$ & $2.5 \pm 1.5$ \\
\hline
\end{tabular}

BCL11A, B-cell CLL/lymphoma 11a; FOX, forkhead box; HOX, homeobox; PCDHGB7, protocadherin $\gamma$ subfamily B, 7. Values are expressed as the mean $\pm \mathrm{SD}$.

Table II. Characteristics of the groups, divided by pathological analysis.

\begin{tabular}{lcc}
\hline Type & $\mathrm{n}$ & Tumor size $(\mathrm{cm})$ \\
\hline Non-infiltrative carcinoma & 45 & $<2$ \\
Invasive carcinoma & 21 & $>2$ \\
Healthy individuals & 16 & 0 \\
\hline
\end{tabular}

treatments, which further resulted in a long survival time of breast cancer patients.

Although various methods for the early-stage diagnosis of breast cancer have been introduced, including contrast-enhanced MRI, it is difficult to differentiate between breast tumors and normal lumps in women with suspected breast cancer (31-33). Molecular diagnosis comprising the detection of high-frequency mutations in breast cancer patients has provided a novel strategy for the early diagnosis of human breast cancer (34). In the present study, the expression levels of six genes, namely Ki-67, BCL11A, FOXC1, HOXD13, PCDHGB7 and her-2, was determined to analyze the risk of breast cancer in a total of 84 patients with suspected breast cancer. The results indicated a significant positive correlation between gene expression levels and the risk of breast cancer, as well as between gene expression levels and tumor size in patients with breast cancer. In the present study, gene expression analysis identified 72 patients with an elevated risk of breast cancer among 84 suspicious patients.

$\mathrm{Ki}-67$ has been regarded as a prognostic marker based on a molecular subtype of breast cancer (35). The present study reported that $\mathrm{Ki}-67$ expression was higher in invasive carcinoma compared with that in non-infiltrative carcinoma. BCL11A is overexpressed in triple-negative breast cancer compared with that in normal mammary epithelial cells (36). In the present study, BCL11A was 12-20-fold increased in tumor biopsy samples in patients with breast cancer compared with that non-malignant breast lumps in healthy individuals. A previous study has indicated that FOXC1 overexpression is a marker of poor response to anthracycline-based adjuvant 
A

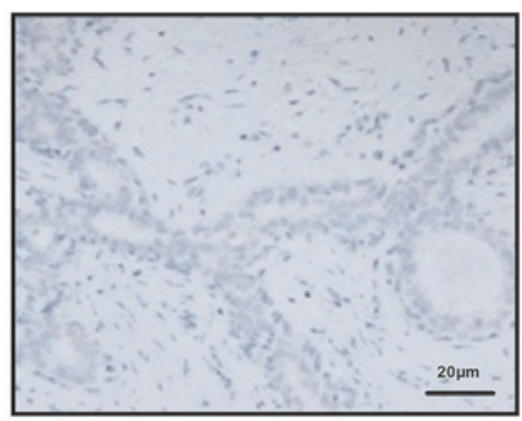

Non-cancerous lump
B

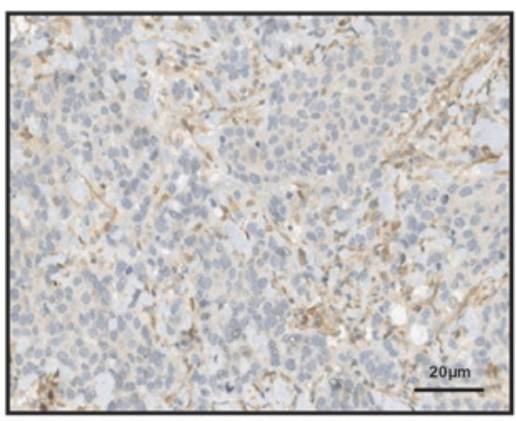

Non-infiltrative carcinoma
C

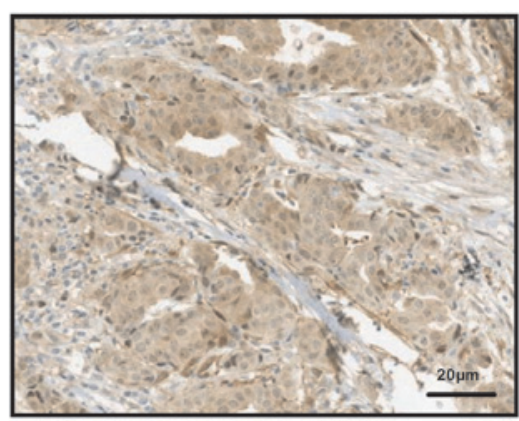

Invasive carcinoma

Figure 2. Pathological analysis of breast cancer tissue. Immunohistochemistry was performed to analyze normal breast and breast cancer tissue (magnification, $\mathrm{x} 40$ ). (A) Non-cancerous lump. (B) Non-infiltrative breast cancer tissue. (C) Invasive breast carcinoma tissue. Scale bar, $20 \mu \mathrm{m}$.

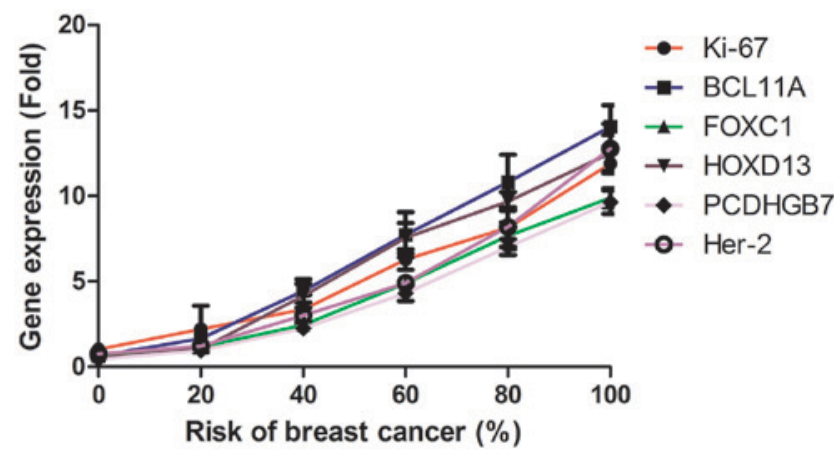

Figure 3. Correlation between gene expression levels of Ki-67, BCL11A, FOXC1, HOXD13, PCDHGB7 and her-2 and the risk of breast cancer. The mean gene expression levels were determined by reverse transcription-quantitative polymerase chain reaction analysis. BCL11A, B-cell CLL/lymphoma 11a; FOX, forkhead box; HOX, homeobox; PCDHGB7, protocadherin $\gamma$ subfamily B7.

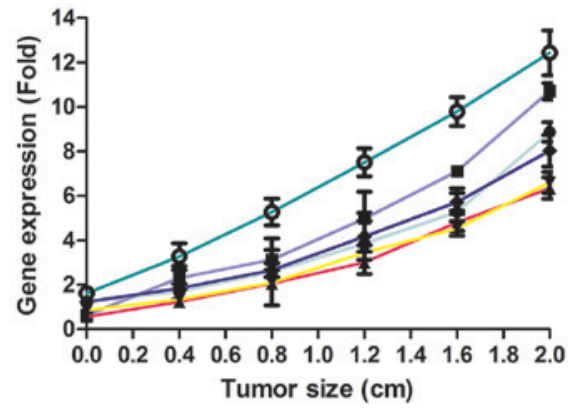

- $\mathrm{Ki}-67$

- BCL11A

\pm FOXC1

- HOXD13

$\bullet$ PCDHGB7

- - Her-2

Figure 4. Correlation between gene expression levels and the tumor size of breast cancer. BCL11A, B-cell CLL/lymphoma 11a; FOX, forkhead box; HOX, homeobox; PCDHGB7, protocadherin $\gamma$ subfamily B7.

chemotherapy in triple-negative breast cancer (37). In addition, the prognostic significance of HOXD13 expression in human breast cancer has been elucidated and a previous study indicated that HOXD13 is a potential prognostic marker for patients with breast cancer (38). Furthermore, quantification of her-2 expression in immunohistochemically-identified biopsies can be used to diagnose breast cancer (39). The present study indicated that the expression levels of BCL11A, FOXC1, HOXD13, PCDHGB7 and her-2 were upregulated

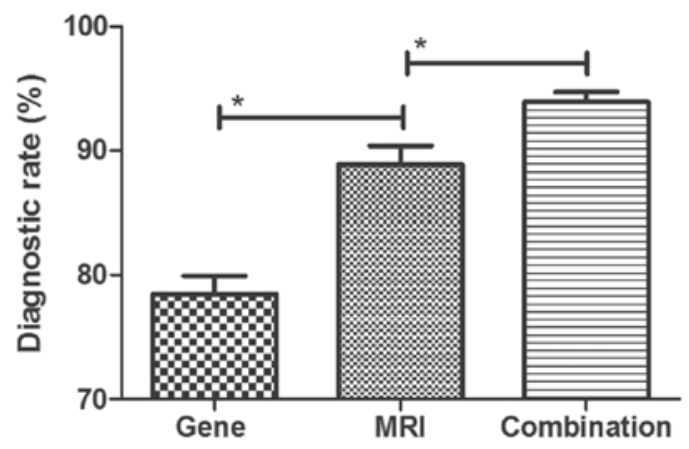

Figure 5. Diagnostic efficacy of a combination of MRI and gene detection for breast cancer patients. ${ }^{*} \mathrm{P}<0.01$. MRI, magnetic resonance imaging.

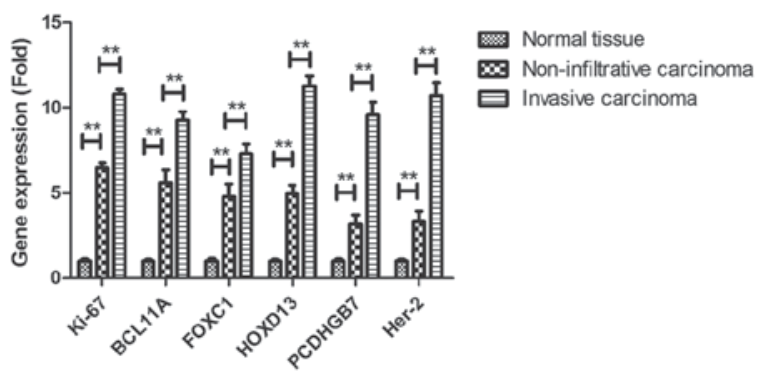

Figure 6. Breast cancer-associated mean gene expression of Ki-67, BCL11A, FOXC1, HOXD13, PCDHGB7 and her-2 levels in non-infiltrative carcinoma and early invasive carcinoma $(\mathrm{n}=8) .{ }^{* *} \mathrm{P}<0.01$.

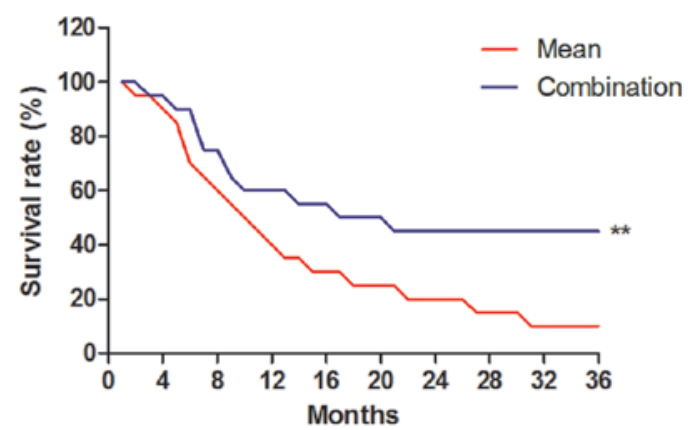

Figure 7. Survival of breast cancer patients diagnosed by magnetic resonance imaging and gene detection. ${ }^{* *} \mathrm{P}<0.01$ vs. mean survival of breast cancer patients. 
in patients with breast cancer. The present study analyzed the diagnostic efficacy of the combination of MRI and detection of gene expression for patients with breast cancer in the early stage.

Studies have indicated that MRI is an accurate diagnostic method for patients with breast cancer $(40,41)$. The present study reported that out of 78 patients in whom a breast lump was detected on MRI, 72 were indicated to have breast cancer according to detection of gene expression. In fact, only 66 patients were confirmed to have breast cancer by pathological analysis. The results of the present study also identified that MRI and detection of gene expression alone are not sufficient for breast cancer diagnosis. The results of the present study also indicated that breast tumor-associated genes were lower expressed in non-infiltrative carcinoma compared with those in in early invasive carcinoma. Of note, the survival time of patients diagnosed by MRI and detection of gene expression was longer than the mean survival of breast cancer patients reported previously (42).

In conclusion, the present study analyzed the efficacy of MRI and detection of gene expression in diagnosing breast cancer patients. It was demonstrated that the detection of the gene expression levels of BCL11A, FOXC1, HOXD13, PCDHGB7 and her-2 may be regarded as an auxiliary method for MRI in diagnosing human breast cancer. However, further studies in large populations of patients with suspected breast cancer are required to determine the combined diagnostic efficacy of MRI and detection of gene expression.

\section{Acknowledgements}

Not applicable.

\section{Funding}

No funding was received.

\section{Availability of data and materials}

The datasets used and/or analyzed during the current study are available from the corresponding author on reasonable request.

\section{Authors' contributions}

DK, RY, LJ analyzed and interpreted the patient data regarding the hematological disease and the transplant. DK performed the histological examination of the kidney, and was a major contributor in writing the manuscript. All authors read and approved the final manuscript.

\section{Ethical approval and consent to participate}

This study was approved by the Ethics Committee of Capital Medical University (Beijing, China). All patients provided written informed consent.

\section{Consent for publication}

Not applicable.

\section{Competing interests}

The authors declare that they have no competing interests.

\section{References}

1. Novik AV, Protsenko SA, Baldueva IA, Ivantsov AO, Nekhaeva TL, Akhaeva ZY, Yanus GA, Iyevleva AG and Imyanitov EN: Vemurafenib-induced progression of breast cancer: A case report and review of the literature. Target Oncol 11: 235-238, 2016.

2. Araki K and Ito Y: A review multigene assays for Clinical Utility in breast cancer. Gan to kagaku ryoho. Gan To Kagaku Ryoho 43: 1332-1340, 2016 (In Japanese).

3. Plourde N, Brown HK, Vigod S and Cobigo V: Contextual factors associated with uptake of breast and cervical cancer screening: A systematic review of the literature. Women Health 56: 906-925, 2016.

4. Post KE and Flanagan J: Web based survivorship interventions for women with breast cancer: An integrative review. Eur J Oncol Nurs 25: 90-99, 2016.

5. Thivya KS, Sakthivel P and Venkata Sai PM: Analysis of framelets for breast cancer diagnosis. Technol Health Care 24: 21-29, 2016.

6. Korkmaz SA, Korkmaz MF and Poyraz M: Diagnosis of breast cancer in light microscopic and mammographic images textures using relative entropy via kernel estimation. Med Biol Eng Comput 54: 561-573, 2016.

7. Zuo X, Chen L, Liu L, Zhang Z, Zhang X, Yu Q, Feng L, Zhao X and Qin T: Identification of a panel of complex autoantigens (LGALS3, PHB2, MUC1, and GK2) in combination with CA15-3 for the diagnosis of early-stage breast cancer. Tumour Biol 37: 1309-1317, 2016.

8. Wang DY, Done SJ, McCready DR, Boerner S, Kulkarni S and Leong WL: A new gene expression signature, the ClinicoMolecular Triad Classification, may improve prediction and prognostication of breast cancer at the time of diagnosis. Breast Cancer Res 13: R92, 2011.

9. Miki Y: Gene expression-based diagnosis of efficacy of chemotherapy for breast cancer. Breast Cancer 17: 97-102, 2010.

10. Richman I, Asch SM, Bendavid E, Bhattacharya J and Owens DK: Breast density notification legislation and breast cancer stage at diagnosis: Early evidence from the SEER registry. J Gen Intern Med 32: 603-609, 2017.

11. Abdoli G, Bottai M, Sandelin K and Moradi T: Breast cancer diagnosis and mortality by tumor stage and migration background in a nationwide cohort study in Sweden. Breast 31: 57-65, 2017.

12. Tang S, Zhou F, Sun Y, Wei L, Zhu S, Yang R, Huang Y and Yang J: CEA in breast ductal secretions as a promising biomarker for the diagnosis of breast cancer: A systematic review and meta-analysis. Breast Cancer 23: 813-819, 2016.

13. Kruger S, Mottaghy FM, Buck AK, Maschke S, Kley H, Frechen D, Wibmer T, Reske SN and Pauls S: Brain metastasis in lung cancer. Comparison of cerebral MRI and 18F-FDG-PET/CT for diagnosis in the initial staging. Nuklearmedizin 50: 101-106, 2011.

14. Peng KQ, Huang ZL, Xie CM, Chen L, Ouyang Y, Zheng QS, Zhang Y, He HQ and Wu PH: Application of dynamic contrast enhancement MRI and post-processing technique for diagnosis of breast cancer. Ai Zheng 28: 549-554, 2009 (In Chinese).

15. Youk JH, Son EJ, Kim EK, Kim JA, Kim MJ, Kwak JY and Lee SM: Diagnosis of breast cancer at dynamic MRI in patients with breast augmentation by paraffin or silicone injection. Clin Radiol 64: 1175-1180, 2009.

16. Morrow M, Waters $\mathrm{J}$ and Morris E: MRI for breast cancer screening, diagnosis, and treatment. Lancet 378: 1804-1811, 2011.

17. Liu T, Cheng T, Xu W, Yan WL, Liu J and Yang HL: A meta-analysis of 18FDG-PET, MRI and bone scintigraphy for diagnosis of bone metastases in patients with breast cancer. Skeletal Radiol 40: 523-531, 2011.

18. Kawashima H, Inokuchi M, Furukawa $\mathrm{H}$ and Kitamura S: Accuracy for a diagnosis of breast cancer spread using 3.0T MRI. Nihon rinsho. Nihon Rinsho 70 (Suppl 7): S5306-S5308, 2012 (In Japanese). 
19. Kwong A, Chen J, Shin VY, Ho JC, Law FB, Au CH, Chan TL, Ma ES and Ford JM: The importance of analysis of long-range rearrangement of BRCA1 and BRCA2 in genetic diagnosis of familial breast cancer. Cancer Genet 208: 448-454, 2015.

20. Woodson AH, Muse KI,Lin H, Jackson M, Mattair DN, Schover L, Woodard T, McKenzie L, Theriault RL, Hortobágyi GN, et al: Breast cancer, BRCA mutations, and attitudes regarding pregnancy and preimplantation genetic diagnosis. Oncologist 19: 797-804, 2014.

21. Derks-Smeets IA, de Die-Smulders CE, Mackens S, van Golde R, Paulussen AD, Dreesen J, Tournaye H, Verdyck P, Tjan-Heijnen VC, Meijer-Hoogeveen M, et al: Hereditary breast and ovarian cancer and reproduction: an observational study on the suitability of preimplantation genetic diagnosis for both asymptomatic carriers and breast cancer survivors. Breast Cancer Res Treat 145: 673-681, 2014.

22. Heacock L, Melsaether AN, Heller SL, Gao Y, Pysarenko KM, Babb JS, Kim SG and Moy L: Evaluation of a known breast cancer using an abbreviated breast MRI protocol: Correlation of imaging characteristics and pathology with lesion detection and conspicuity. Eur J Radiol 85: 815-823, 2016.

23. Ethier JL and Amir E: The Role of the 21-gene recurrence score in breast cancer treatment. Mol Diagn Ther 20: 307-313, 2016.

24. Hoogland AM, Bottcher R, Verhoef E, Jenster G and van Leenders GJ: Gene-expression analysis of gleason grade 3 tumor glands embedded in low- and high-risk prostate cancer. Oncotarget 7: 37846-37856, 2016.

25. Livak KJ and Schmittgen TD: Analysis of relative gene expression data using real-time quantitative PCR and the 2(-Delta Delta C(T)) method. Methods 25: 402-408, 2001.

26. Printz C: New AJCC cancer staging manual reflects changes in cancer knowledge. Cancer 116: 2-3, 2010.

27. Park HS, Park S, Kim JH, Lee JH, Choi SY, Park BW and Lee KS: Clinicopathologic features and outcomes of metaplastic breast carcinoma: Comparison with invasive ductal carcinoma of the breast. Yonsei Med J 51: 864-869, 2010.

28. Cho JH, Park JM, Park HS, Park S, Kim SI and Park BW: Oncologic safety of breast-conserving surgery compared to mastectomy in patients receiving neoadjuvant chemotherapy for locally advanced breast cancer. J Surg Oncol 108: 531-536, 2013.

29. Telegrafo M, Rella L, Stabile Ianora AA, Angelelli G and Moschetta M: Breast MRI background parenchymal enhancement (BPE) correlates with the risk of breast cancer. Magn Reson Imaging 34: 173-176, 2016.

30. Masood S, El-Gabry E, Zhang C and Wang Z: The potential of identification of a malignancy-associated biomarker in breast cancer diagnosis and research: hTERT gene DNA methylation. Diagn Cytopathol 44: 670-675, 2016.
31. Yoon H, Yoon D, Yun M, Choi JS, Park VY, Kim EK, Jeong J, Koo JS, Yoon JH, Moon HJ, et al: Metabolomics of Breast Cancer Using High-Resolution Magic Angle Spinning Magnetic Resonance Spectroscopy: Correlations with 18F-FDG Positron Emission Tomography-Computed Tomography, Dynamic Contrast-Enhanced and Diffusion-Weighted Imaging MRI PLoS One 11: e0159949, 2016.

32. Wu S, Berg WA, Zuley ML, Kurland BF, Jankowitz RC, Nishikawa R, Gur D and Sumkin JH: Breast MRI contrast enhancement kinetics of normal parenchyma correlate with presence of breast cancer. Breast Cancer Res 18: 76, 2016.

33. Chung MT, Lourenco AP and Mainiero MB: Screening Breast MRI in Women with a Personal History of Breast Cancer. Breast J 22: 252-253, 2016.

34. Mannan AU, Singh J, Lakshmikeshava R, Thota N, Singh S, Sowmya TS, Mishra A, Sinha A, Deshwal S, Soni MR, et al: Detection of high frequency of mutations in a breast and/or ovarian cancer cohort: Implications of embracing a multi-gene panel in molecular diagnosis in India. J Hum Genet 61: 515-522, 2016.

35. Soliman NA and Yussif SM: Ki-67 as a prognostic marker according to breast cancer molecular subtype. Cancer Biol Med 13: 496-504, 2016.

36. Khaled WT, Choon Lee S, Stingl J, Chen X, RazaAli H, Rueda OM, Hadi F, Wang J, Yu Y, Chin SF, et al: BCL11A is a triple-negative breast cancer gene with critical functions in stem and progenitor cells. Nat Commun 6: 5987, 2015.

37. Xu YL, Yao R, Li J, Zhou YD, Mao F, Pan B and Sun Q: FOXC1 overexpression is a marker of poor response to anthracycline-based adjuvant chemotherapy in sporadic triple-negative breast cancer. Cancer Chemother Pharmacol 79: 1205-1213, 2017.

38. Zhong ZB, Shan M, Qian C, Liu T, Shi QY, Wang J, Liu Y, Liu Y, Huang YX and Pang D: Prognostic significance of HOXD13 expression in human breast cancer. Int J Clin Exp Pathol 8: 11407-11413, 2015

39. Mendoza G, Portillo A and Olmos-Soto J: Accurate breast cancer diagnosis through real-time PCR her-2 gene quantification using immunohistochemically-identified biopsies. Oncol Lett 5: 295-298, 2013.

40. Swayampakula AK, Dillis C and Abraham J: Role of MRI in screening, diagnosis and management of breast cancer. Expert Rev Anticancer Ther 8: 811-817, 2008.

41. Tozaki M: Diagnosis of breast cancer: MDCT versus MRI. Breast Cancer 15: 205-211, 2008

42. Schwentner L, Wolters R, Wischnewsky M, Kreienberg R and Wockel A: Survival of patients with bilateral versus unilateral breast cancer and impact of guideline adherent adjuvant treatment: A multi-centre cohort study of 5292 patients. Breast 21: 171-177, 2012. 